\title{
OPTIMAL PORTFOLIOS FOR FINANCIAL MARKETS WITH WISHART VOLATILITY
}

\author{
NICOLE BÄUERLE* AND ZEJING LI ${ }^{\ddagger}$
}

\begin{abstract}
We consider a multi asset financial market with stochastic volatility modeled by a Wishart process. This is an extension of the one-dimensional Heston model. Within this framework we study the problem of maximizing the expected utility of terminal wealth for power and logarithmic utility. We apply the usual stochastic control approach and obtain explicitly the optimal portfolio strategy and the value function in some parameter settings. In particular when the drift of the assets is a linear function of the volatility matrix. In this case the affine structure of the model can be exploited. In some cases we obtain a Feynman-Kac representation of the candidate value function. Though the approach we use is quite standard, the hard part is indeed to identify when the solution of the HJB equation is finite. This involves a couple of matrix analytic arguments. In a numerical study we discuss the influence of the investors' risk aversion on the hedging demand.
\end{abstract}

Key words: Wishart Process, Portfolio Problem, CRRA Utility, Stochastic Control, HJB Equation, Matrix Exponential. AMS SUBJECT ClASSIFICATIONS: 93E20, 91G80, 91G10.

\section{INTRODUCTION}

Asset price models need a stochastic volatility in order to produce the 'smile effect' between the implied volatility and the strike price of a call option or to reproduce the 'leverage effect', i.e. that past returns are negatively correlated with future volatilities. To cover these aspects, a lot of one-factor stochastic volatility models have been introduced in the course of time, among them the popular Heston model (see Heston (1993)). However, recent empirical studies show that even in a single asset model it might be reasonable to model the volatility by at least two factors (see e.g. Cont \& da Fonseca (2002)). Moreover it is often necessary to model a complete portfolio of assets. Hence in recent times multivariate extensions of the Heston model have been proposed. They all build on the matrix Wishart process for the volatility which has been introduced in $\mathrm{Bru}(1991)$. It is a direct multivariate extension of the Cox-Ingersoll-Ross model and has been extended and used for financial applications by e.g. Gourieroux \& Sufana (2003, 2004); Da Fonseca et al. (2007, 2008); Buraschi et al. (2010); Muhle-Karbe et al. (2012). While these papers consider option pricing, hedging, credit risk and term structure models, we will investigate portfolio optimization problems. More precisely we consider the classical problem of maximizing the expected utility of terminal wealth in a multi asset Wishart volatility market for power and logarithmic utility. During the work on this project we became aware that Hata \& Sekine (2011) also consider portfolio optimization problems in a similar setup with the risksensitive criterion. However their methods are different and they also focus on different aspects as we will explain below. Another recent work is Richter $(2012)$ where solutions of BSDEs in such a setting are considered and applied to indifference pricing. There also the exponential utility is dealt with. In Buraschi et al. (2010) a model with two assets and two-dimensional Wishart process is considered and the hedging demand for a power utility problem is treated. Besides these works, to the best of our knowledge, there are no others yet which deal with optimization problems in this financial market. Moreover, all these papers consider only financial markets where the process of volatility and stochastic logarithm of the asset has an affine differential w.r.t. the volatility. We call this the affine case. In Kallsen \& Muhle-Karbe (2010) the authors 
consider a general semimartingale market which is again affine. They consider portfolio problems with power utility but only in the single asset framework.

In this paper we use the classical stochastic control approach and solve the portfolio optimization problem with the help of the Hamilton-Jacobi-Bellman (HJB) equation. However it turns out that this is here indeed a non-trivial task due to rather complicated computations. Moreover some interesting aspects occur in the sense that in some parameter settings the problem is rather easy and in some not. We already know from Kraft (2005) (see also Kallsen \& Muhle-Karbe (2010) and Liu (2007)) that in the one-dimensional Heston model which is included as a special case, there are parameter setings where the value function is not finite and thus the stochastic control approach breaks down. See also Korn \& Kraft (2004) for a warning word in this direction. It will turn out that in the affine case (i.e. when the drift of the stocks is a linear function of the Wishart process, which is assumed in most models and also in the Heston model), there are parameter settings where the value function is finite and can be computed explicitly. In this case fortunately we can show that the optimal value function is a Laplace transform of the Wishart volatility process and we can use results in Gnoatto \& Grasselli (2012) where such expressions have been computed. The affine structure of the process is here exploited. Still the impression remains that this is a very special case because the corresponding optimal portfolio strategy is completely deterministic and hence measurable w.r.t. the initial information. We also identify another situation with special correlation between the Brownian motions which drive the asset dynamics and those which drive the volatility dynamics and a special $Q$ matrix, however general drift, where the HJB equation boils down to a linear partial differential equation and where we have at least a candidate for the value function via a Feynman-Kac representation formula. In particular the case of uncorrelated Brownian motions belongs to this category.

In Hata \& Sekine (2011) the authors solve a risk-sensitive portfolio problem in an affine Wishart-volatility model. While doing this, they also solve the power utility problem. However they use the risk sensitive approach and do a change of measure first, before they set up the HJB equation. Moreover they do not get a closed form solution like we do and also do not discuss the cases where the value function is infinite. On the other side they also tackle infinite horizon problems.

The outline of our paper is as follows: In section 2 we introduce the multi asset financial market where volatility is modeled by a Wishart process and state the optimization problem. In section 3 we derive the associated HJB equation. The next section is then dedicated to finding solutions of this HJB equation. We divide this section into two parts. In the first part we consider a general asset drift, but specific correlations and in the second part we treat the affine model where we derive an explicit solution. In section 5 we verify that this solution in the affine model is indeed the value function of our portfolio problem. In section 6 we briefly discuss the case of logarithmic utility function and in section 7 we consider some numerical examples and discuss the hedging demand in a two-dimensional setting. The appendix contains some of the proofs and auxiliary results.

\section{The Wishart Volatility Market}

It is well-known that the classical standard Black-Scholes model is not flexible enough to create the simile effect, nor does it satisfy the leverage effect either.

To cover these shortages of the standard Black-Scholes model, Gourieroux \& Sufana (2004) have presented a multivariate Wishart stochastic volatility. The model introduced below possesses a generalized drift compared with Gourieroux \& Sufana (2004). It is an extension of the one-dimensional Heston model.

In our model the market consists of one riskfree asset with price process $\left(S_{t}^{0}\right)_{t>0}$ and $d$ risky assets. The constant riskfree rate is $r \geq 0$ and the dynamic of the riskfree asset is

$$
d S_{t}^{0}=S_{t}^{0} r d t, S_{t}^{0}=1
$$


We denote by $\left(S_{t, i}\right)_{t \geq 0}, 1 \leq i \leq d$ the price processes of the $d$ risky assets and by $\left(S_{t}\right)_{t \geq 0}=$ $\left(S_{t, 1}, \ldots, S_{t, d}\right)_{t \geq 0}$ the vector process. The return of $\left(S_{t}\right)_{t \geq 0}$ owns a Wishart stochastic volatility $\left(\Sigma_{t}\right)_{t \geq 0}$. The joint dynamics of $\left(S_{t}\right)_{t \geq 0}$ and $\left(\Sigma_{t}\right)_{t \geq 0}$ are given by the following (vector-matrix-) stochastic differential system:

$$
\begin{aligned}
& d S_{t}=\operatorname{diag}\left(S_{t}\right)\left(B\left(\Sigma_{t}\right) d t+\Sigma_{t}^{1 / 2} d W_{t}^{S}\right), \\
& d \Sigma_{t}=\left(N N^{T}+M \Sigma_{t}+\Sigma_{t} M^{T}\right) d t+\Sigma_{t}^{1 / 2} d W_{t}^{\sigma} Q+Q^{T}\left(d W_{t}^{\sigma}\right)^{T} \Sigma_{t}^{1 / 2},
\end{aligned}
$$

where $\left(W_{t}^{S}\right)_{t \geq 0}$, is a $d$ dimensional Brownian motion vector and $\left(W_{t}^{\sigma}\right)_{t \geq 0}$ is a $d \times d$ Brownian motion matrix respectively. All processes are defined on a common probability space $(\Omega, \mathcal{F}, \mathbb{P})$. In what follows, $\left(\mathcal{F}_{t}\right)_{t \geq 0}$ denotes the corresponding Brownian filtration. The entries between $\left(W_{t}^{S}\right)_{t \geq 0}$ and $\left(W_{t}^{\sigma}\right)_{t \geq 0}$ can be correlated. We assume that $d\left\langle W_{t, k}^{S}, W_{t, i j}^{\sigma}\right\rangle=\rho_{k, i j} d t$ for $1 \leq$ $k, i, j \leq d$. The matrix $\operatorname{diag}\left(S_{t}\right)$ is a diagonal matrix with entries $S_{t, 1}, \ldots, S_{t, d}$ on the diagonal.

Further $N, \mathrm{M}, \mathrm{Q}$ are $d \times d$ matrices with $N \in G L_{d}(\mathbb{R})$ the set of real invertible matrices of dimension $d \times d$. We also assume that $N N^{T} \succeq(d+1) Q^{T} Q$ (where $A \succeq B$ means that $A-B$ is positive semidefinite) which according to Theorem 2.2 in Mayerhofer et al. (2011) implies that 2.2 has a unique global strong solution on $S_{d}^{+}(\mathbb{R})$ which is the set of symmetric positive definite matrices of dimension $d \times d$. As usual we denote for $\Sigma \in S_{d}^{+}(\mathbb{R})$ by $\Sigma^{1 / 2}$ the unique matrix $A \in S_{d}^{+}(\mathbb{R})$ such that $A^{2}=\Sigma$. The function $B: S_{d}^{+}(\mathbb{R}) \rightarrow \mathbb{R}^{d}$ is measurable and will be specified later.

In what follows we will assume for the correlation coefficients $\rho_{k, i j}, 1 \leq k, i, j \leq d$ between the Brownian motions that $\rho_{k, i j}=0$ for $k \neq i$ and $\rho_{k, k j}=: \rho_{j}$ is independent of $k$. In particular we denote $\boldsymbol{\rho}=\left(\rho_{1}, \ldots, \rho_{d}\right)^{T}$. This means if $\left(B_{t}\right)$ is another $d$-dimensional Brownian motion vector independent from $\left(W_{t}^{\sigma}\right)$, we assume that

$$
W_{t}^{S} \stackrel{d}{=} \sqrt{1-\boldsymbol{\rho}^{T} \boldsymbol{\rho}} B_{t}+W_{t}^{\sigma} \boldsymbol{\rho} .
$$

Example 2.1. The Wishart stochastic volatility model can be regarded as an extension of the Heston model to the multidimensional case. Recall that the one-dimensional asset return process $\left(S_{t}\right)_{t \geq 0}$ in the Heston model is determined by the stochastic process:

$$
d S_{t}=S_{t}\left(\mu+\lambda Z_{t}\right) d t+\sqrt{Z_{t}} d W_{t}^{S},
$$

whereas the volatility process $\left(Z_{t}\right)_{t \geq 0}$ follows a Cox-Ingersoll-Ross process:

$$
d Z_{t}=\kappa\left(\theta-Z_{t}\right) d t+\xi \sqrt{Z_{t}} d W_{t}^{Z},
$$

where $\left(W_{t}^{S}\right)_{t \geq 0},\left(W_{t}^{Z}\right)_{t \geq 0}$ are Brownian motions with correlation $\rho$ and $\mu, \kappa, \theta$ and $\xi$ are suitable constants in $\mathbb{R}$. One can easily see that the dynamics above are specifications of (2.1) and (2.2) in the one-dimensional case.

We assume now that an agent can invest into this financial market and define the portfolio strategy process $\left(\pi_{t}\right)_{t \geq 0}$ as an $\mathbb{R}^{d}$-valued progressively measurable process with respect to $\left(\mathcal{F}_{t}\right)_{t \geq 0}$ where $\pi_{t}=\left(\pi_{t, 1}, \ldots, \pi_{t, d}\right)^{T}$ and $\pi_{t, k}$ represents the proportion of wealth invested into stock $k$ at time $t$. Obviously $\pi_{t}^{0}:=1-\pi_{t}^{T} \mathbf{1}$ is the proportion of wealth invested in the bond where $\mathbf{1}=(1, \ldots, 1)^{T} \in \mathbb{R}^{d}$. Under a fixed portfolio strategy $\left(\pi_{t}\right)_{t \geq 0}$, the portfolio wealth process $\left(X_{t}^{\pi}\right)_{t \geq 0}$ owns the following dynamic:

$$
d X_{t}^{\pi}=X_{t}^{\pi} \pi_{t}^{T} \frac{d S_{t}}{S_{t}}+X^{\pi} \pi_{t}^{0} \frac{d S_{t}^{0}}{S_{t}^{0}}
$$

Applying the dynamic (2.1) yields

$$
\frac{d X_{t}^{\pi}}{X_{t}^{\pi}}=\left(\pi_{t}^{T}\left(B\left(\Sigma_{t}\right)-\mathbf{r}\right)+r\right) d t+\pi_{t}^{T} \Sigma_{t}^{1 / 2} d W_{t}^{S}
$$


with $X_{0}^{\pi}=x_{0}$ and $\mathbf{r}=(r, \ldots, r)^{T} \in \mathbb{R}^{d}$. We consider only portfolio strategies where (2.4) has a unique strong solution and call them admissible. The solution of the portfolio wealth process $\left(X_{t}^{\pi}\right)$ is given as follows:

$$
X_{T}^{\pi}=x_{0} \exp \left(\int_{0}^{T}\left[\pi_{s}^{T}\left(B\left(\Sigma_{s}\right)-\mathbf{r}\right)+r-\frac{1}{2}\left\|\pi_{s}^{T} \Sigma_{s}^{1 / 2}\right\|_{2}^{2}\right] d s+\int_{0}^{T} \pi_{s}^{T} \Sigma_{s}^{1 / 2} d W_{s}^{S}\right) .
$$

Now denote by $U: \mathbb{R}_{+} \rightarrow \mathbb{R}$ a (strictly increasing, strictly concave) utility function. We want to solve the classical problem of maximizing expected utility of terminal wealth for power and logarithmic utility. The value function of the optimization problem reads as

$$
V(t, x, \Sigma)=\sup _{\pi} \mathbb{E}^{t, x, \Sigma}\left[U\left(X_{T}^{\pi}\right)\right], \quad x>0, \Sigma \in S_{d}^{+}(\mathbb{R}), t \in[0, T]
$$

where $\mathbb{E}^{t, x, \Sigma}$ is the expectation w.r.t. the conditional distribution $X_{t}=x, \Sigma_{t}=\Sigma$ and where the supremum is taken over all admissible portfolio strategies. We will follow the usual way using stochastic control, i.e. we will first derive the HJB equation, then find a solution and finally verify it. However, the challenge here is to identify parameter cases where indeed the value function is finite. As pointed out in Kraft (2005) and Korn \& Kraft (2004) this is an important issue and in this case a non-trivial task.

\section{The HJB Equation and its Transformations}

In what follows we assume that $U(x)=\frac{1}{\gamma} x^{\gamma}$ for $\gamma<1, \gamma \neq 0$. The formal derivation of the HJB equation is as follows: Since the process $\left(V\left(t, X_{t}^{\pi}, \Sigma_{t}\right)\right)_{t \geq 0}$ is a supermartingale under any admissible portfolio strategy $\pi$ and a martingale under the optimal one, the drift of the process which is derived using the Itô-Doeblin formula has to be zero when maximized over all portfolio allocations. In order to apply the Itô-Doeblin formula we have to compute the quadratic variation and covariation of $\left(X_{t}^{\pi}\right)$ and $\left(\Sigma_{t}\right)$ :

$$
\begin{aligned}
d\left\langle\Sigma_{l k}, \Sigma_{p q}\right\rangle_{t} & =\left[\Sigma_{l p}(t)\left(Q^{T} Q\right)_{k q}+\Sigma_{p k}(t)\left(Q^{T} Q\right)_{q l}+\Sigma_{l q}(t)\left(Q^{T} Q\right)_{k p}+\Sigma_{k q}(t)\left(Q^{T} Q\right)_{l p}\right] d t \\
d\left\langle\Sigma_{l k}, X^{\pi}\right\rangle_{t} & =X_{t}^{\pi}\left[\left(\pi_{t}^{T} \Sigma_{t}\right)_{l}\left(Q^{T} \boldsymbol{\rho}\right)_{k}+\left(\pi_{t}^{T} \Sigma_{t}\right)_{k}\left(Q^{T} \boldsymbol{\rho}\right)_{l}\right] d t \\
d\left\langle X^{\pi}\right\rangle_{t} & =\left(X_{t}^{\pi}\right)^{2}\left[\pi_{t}^{T} \Sigma_{t} \pi_{t}\right] d t .
\end{aligned}
$$

We will also denote by $G_{t}$ and $G_{x}$ the partial derivative w.r.t. $t$ and $x$ and we denote the operator matrix

$$
\nabla:=\left(\frac{\partial}{\partial \Sigma_{i j}}\right)_{1 \leq i, j \leq d} .
$$

Thus, a candidate $G(t, x, \Sigma) \in C^{1,2,2}\left([0, T] \times \mathbb{R}_{+} \times S_{d}^{+}(\mathbb{R})\right)$ for the value function should satisfy the HJB equation:

$$
\begin{aligned}
& 0=G_{t}+\operatorname{Tr}\left(\left(N N^{T}+M \Sigma+\Sigma M^{T}\right) \nabla G\right)+r x G_{x}+\frac{1}{2} \operatorname{Tr}\left(\Sigma\left(\nabla+\nabla^{T}\right)\left(Q^{T} Q\right)\left(\nabla^{T}+\nabla\right)\right) G+ \\
& +\sup _{u \in \mathbb{R}^{d}}\left\{x u^{T}(B-\mathbf{r}) G_{x}+\frac{1}{2} x^{2} u^{T} \Sigma u G_{x x}+x u^{T} \Sigma\left(\nabla+\nabla^{T}\right) G_{x} Q^{T} \boldsymbol{\rho}\right\},
\end{aligned}
$$

with terminal condition $G(T, x, \Sigma)=\frac{1}{\gamma} x^{\gamma}$. When we use the usual Ansatz $G(t, x, \Sigma)=\frac{x^{\gamma}}{\gamma} g(t, \Sigma)$ with $g>0$ and plug in the expressions above we end up with the HJB equation

$$
\begin{aligned}
& \frac{1}{\gamma}\left(g_{t}+\frac{1}{2} \operatorname{Tr}\left(\Sigma\left(\nabla+\nabla^{T}\right)\left(Q^{T} Q\right)\left(\nabla^{T}+\nabla\right)\right) g+\operatorname{Tr}\left(\left(N N^{T}+M \Sigma+\Sigma M^{T}\right) \nabla g\right)\right)+r g \\
& +\sup _{u \in \mathbb{R}^{d}}\left\{u^{T}(B-\mathbf{r}) g+\frac{\gamma-1}{2} u^{T} \Sigma u g+u^{T} \Sigma\left(\nabla+\nabla^{T}\right) g Q^{T} \boldsymbol{\rho}\right\}=0 .
\end{aligned}
$$


Obviously a maximizer of this HJB equation is given by

$$
\pi^{*}(t, \Sigma)=\Sigma^{-1}\left(\frac{(B(\Sigma)-\mathbf{r}) g(t, \Sigma)+\Sigma\left(\nabla+\nabla^{T}\right) g(t, \Sigma) Q^{T} \boldsymbol{\rho}}{(1-\gamma) g(t, \Sigma)}\right), \quad \Sigma \in S_{d}^{+}(\mathbb{R}), 0 \leq t \leq T .
$$

Plugging the maximum point into the HJB equation we arrive at

$$
\begin{aligned}
& g_{t}+\frac{1}{2} \operatorname{Tr}\left(\Sigma\left(\nabla+\nabla^{T}\right)\left(Q^{T} Q\right)\left(\nabla^{T}+\nabla\right)\right) g++\operatorname{Tr}\left(\left(N N^{T}+M \Sigma+\Sigma M^{T}\right) \nabla g\right)+\gamma r g \\
& +\frac{\gamma}{2(1-\gamma) g}\left((B-\mathbf{r})^{T} \Sigma^{-1}(B-\mathbf{r}) g^{2}+2 \boldsymbol{\rho}^{T} Q\left(\nabla+\nabla^{T}\right) g(B-\mathbf{r}) g\right. \\
& \left.+\boldsymbol{\rho}^{T} Q\left(\nabla+\nabla^{T}\right) g \Sigma\left(\nabla+\nabla^{T}\right) g Q^{T} \boldsymbol{\rho}\right)=0 .
\end{aligned}
$$

In the next section we will solve (3.4) under further conditions on the model parameters. Note in particular that $B=B(\Sigma)$ is up to now an arbitrary (measurable) function of $\Sigma$. In Section 4 we will consider the following two cases:

(i) Case 1: $B(\Sigma)$ is general and

$$
\boldsymbol{\rho}=\hat{\rho} \mathbf{1} \quad \text { and } \quad Q_{i j}=c_{i} Q_{1 j} \quad c_{i} \in \mathbb{R}, \quad 2 \leq i \leq d, c_{1}:=1,
$$

i.e. the correlations are the same and the rows of $Q$ are multiples of the first one. Technically this condition implies that under the transformation $g=h^{\delta}$ for a suitable $\delta$ the last two terms in (3.4) at least partially cancel and there remains a linear partial differential equation. whose solution can be represented by a Feynman-Kac formula.

(ii) Case 2: $B(\Sigma)-\mathbf{r}=\Sigma \mathbf{v}$ for a $\mathbf{v} \in \mathbb{R}^{d}$. This assumption implies a constant market price $\mathbf{v}$ of variance-covariance risk. It is also used in the one-dimensional Heston model (see Example 2.1). In this case it is possible to derive an explicit solution. In some parameter cases one can show that the solution is finite. The key property that makes this case solvable is the fact that the Wishart process $\left(Z_{t}\right)$ is an affine process and the value function of the problem can be interpreted as a Laplace transform of $\left(\Sigma_{t}\right)$.

\section{Solutions of the HJB Equation}

4.1. A Candidate for general Drift. In this section we consider case (i), so let us assume (3.5). This implies

$$
Q^{T} \boldsymbol{\rho} \boldsymbol{\rho}^{T} Q=\hat{\rho}^{2} Q^{T} \mathbf{1 1}^{T} Q=\rho^{2} Q^{T} Q \text { with } \rho^{2}=\hat{\rho}^{2} \frac{\left(\sum_{i=1}^{d} c_{i}\right)^{2}}{\sum_{i=1}^{d} c_{i}^{2}} .
$$

We use the further transformation

$$
g(t, \Sigma)=h(t, \Sigma)^{\delta}
$$

with

$$
\delta:=\frac{(1-\gamma)}{(1-\gamma)+\gamma \rho^{2}} .
$$

In this case equation (3.4) reduces to the linear partial differential equation

$$
\begin{aligned}
& h_{t}+\operatorname{Tr}\left(\Sigma\left(\nabla+\nabla^{T}\right)\left(Q^{T} Q\right)\left(\nabla^{T}+\nabla\right)\right) h+ \\
& \operatorname{Tr}\left(\left(N N^{T}+M \Sigma+\Sigma M^{T}+H\right) \nabla h\right)+\left(\frac{\gamma r}{\delta}+\frac{\gamma}{2(1-\gamma) \delta}(B-\mathbf{r})^{T} \Sigma^{-1}(B-\mathbf{r})\right) h=0
\end{aligned}
$$

with terminal condition $h(T, \Sigma)=1$ and matrix

$$
H:=\frac{\gamma}{(1-\gamma)}\left(Q^{T} \boldsymbol{\rho}(B-\mathbf{r})^{T}+(B-\mathbf{r}) \boldsymbol{\rho}^{T} Q\right) .
$$

This specific transformation has been used before by Zariphopoulou (2001) and in particular by Kraft (2005) in the one-dimensional Heston model and in Rieder \& Bäuerle (2005) in a model with partial observation. Here we get exactly the same $\delta$ as in Kraft (2005), p. 305. 
It is now possible to formally derive a solution via a Feynman-Kac formula. So far we worked under the physical measure $\mathbb{P}$. We now denote a new measure by $\tilde{\mathbb{P}}$ associated with the following Radon-Nikodym derivative:

$$
Z_{t}:=\left.\frac{d \tilde{\mathbb{P}}}{d \mathbb{P}}\right|_{\mathcal{F}_{t}}=\exp \left(\int_{t}^{T} \operatorname{Tr}\left(\theta\left(\Sigma_{s}\right)^{T} d W_{s}^{\sigma}\right)-\frac{1}{2} \int_{t}^{T}\left\|\theta\left(\Sigma_{s}\right)\right\|^{2} d s\right)
$$

with

$$
\theta(\Sigma):=\frac{\gamma}{1-\gamma}\left(\Sigma^{1 / 2}\right)^{-1}(B(\Sigma)-\mathbf{r}) \boldsymbol{\rho}^{T}, \quad \Sigma \in S_{d}^{+}(\mathbb{R})
$$

For a $d \times d$ matrix $A$ we define by $\|A\|^{2}=\sum_{i, j} a_{i j}^{2}$ the Frobenius norm. Then, we obtain the following theorem which is proved in the appendix:

Theorem 4.1. If the Radon-Nikodym derivative $\left(Z_{t}\right)$ is an $\left(\mathcal{F}_{t}\right)$-martingale and if

$$
\tilde{h}(t, \Sigma):=\tilde{\mathbb{E}}^{\Sigma, t}\left[\exp \left(\int_{t}^{T}\left(\frac{\gamma r}{\delta}+\frac{\gamma}{2(1-\gamma) \delta}\left(B\left(\Sigma_{s}\right)-\mathbf{r}\right)^{T} \Sigma_{s}^{-1}\left(B\left(\Sigma_{s}\right)-\mathbf{r}\right)\right) d s\right)\right] \in C^{1,2}(O)
$$

with $O=[0, T] \times S_{d}^{+}(\mathbb{R})$, then the solution $h$ of 4.3 if it exists, has the Feynman-Kac representation $\tilde{h}$, where $\tilde{\mathbb{E}}$ denotes the expectation under $\tilde{\mathbb{P}}$.

Hence we have a candidate solution for the stochastic control problem. However for a general function $B$ it is difficult to compute the expectation or verify the solution. In the next subsection we consider the special case of a linear function $B$ where we get an explicit solution.

4.2. The Linear Drift Case. In this subsection, we consider case (ii), i.e. the drift coefficient $B(\Sigma)$ of $\left(S_{t}\right)_{t \geq 0}$ satisfies $B(\Sigma)-\mathbf{r}=\Sigma \mathbf{v}$ for a $\mathbf{v} \in \mathbb{R}^{d}$. The asset dynamic $\left(S_{t}\right)_{t \geq 0}$ can now be written as

$$
d S_{t}=\operatorname{diag}\left(S_{t}\right)\left(\left(\mathbf{r}+\Sigma_{t} \mathbf{v}\right) d t+\Sigma_{t}^{1 / 2} d W_{t}^{S}\right) .
$$

The key property that makes this case solvable is the fact that the Wishart process is an affine process and its Laplace transform can be computed (see e.g. Gnoatto \& Grasselli (2012)). We will later see that under the optimal strategy the expected utility of terminal wealth exactly reduces to a Laplace transform of the Wishart process. The partial differential equation in (3.4) now reads

$$
\begin{aligned}
& g_{t}+\frac{1}{2} \operatorname{Tr}\left(\Sigma\left(\nabla+\nabla^{T}\right)\left(Q^{T} Q\right)\left(\nabla^{T}+\nabla\right)\right) g+\operatorname{Tr}\left(\left(N N^{T}+M \Sigma+\Sigma M^{T}\right) \nabla g\right)+\gamma r g \\
& +\frac{\gamma}{2(1-\gamma) g}\left(\mathbf{v}^{T} \Sigma \mathbf{v} g^{2}+2 \boldsymbol{\rho}^{T} Q\left(\nabla^{T}+\nabla\right) g \Sigma \mathbf{v} g+\boldsymbol{\rho}^{T} Q\left(\nabla^{T}+\nabla\right) g \Sigma\left(\nabla+\nabla^{T}\right) g Q^{T} \boldsymbol{\rho}\right)=0
\end{aligned}
$$

with $g(T, \Sigma)=1$.

In the next theorem $S_{d}(\mathbb{R})$ denotes the set of symmetric real matrices of dimension $d$. The proof can again be found in the appendix.

Theorem 4.2. The partial differential equation (4.7) with boundary condition $g(T, \Sigma)=1$ possesses the following solution in case the expressions are finite:

$$
g(t, \Sigma)=\exp (\phi(T-t)+\operatorname{Tr}[\psi(T-t) \Sigma]),
$$

where $\phi(t) \in \mathbb{R}$ and $\psi(t) \in S_{d}(\mathbb{R})$ for $t \in[0, T]$ are solutions of the following Riccati equations system:

$$
\begin{gathered}
\psi_{t}(t)=\psi(t) \tilde{M}+\tilde{M}^{T} \psi(t)+2 \psi(t) \tilde{Q}^{T} \tilde{Q} \psi(t)+\tilde{\Gamma}, \\
\phi_{t}(t)=\operatorname{Tr}\left[\psi(t) N N^{T}\right]+\gamma r
\end{gathered}
$$

with

$$
\tilde{M}=M+\frac{\gamma}{(1-\gamma)} Q^{T} \boldsymbol{\rho} \mathbf{v}^{T}, \quad \tilde{Q}^{T} \tilde{Q}=Q^{T} Q+\frac{\gamma}{(1-\gamma)} Q^{T} \boldsymbol{\rho} \boldsymbol{\rho}^{T} Q, \quad \tilde{\Gamma}=\frac{\gamma}{2(1-\gamma)} \mathbf{v} \mathbf{v}^{T}
$$


and the initial conditions: $\psi(0)=\mathbf{0} \in S_{d}(\mathbb{R}), \phi(0)=0 \in \mathbb{R}$.

Note that equation (4.10) is not a problem because $\phi$ can simply be obtained by integrating the right hand side. The Riccati equations (4.9) have a finite solution in some parameter settings and there are different ways to compute a solution. For example it is possible to use a linearization of the matrix Riccati ODE (see Gnoatto \& Grasselli (2012) for details). In this case one obtains with Proposition 11 in Gnoatto \& Grasselli (2012):

Proposition 4.3. In case a finite solution of 4.9 exists for $t \in[0, T]$ with $\psi(0)=0$, it is given by

$$
\psi(t)=\psi_{22}(t)^{-1} \psi_{21}(t)
$$

where

$$
\left(\begin{array}{ll}
\psi_{11}(t) & \psi_{12}(t) \\
\psi_{21}(t) & \psi_{22}(t)
\end{array}\right)=\exp \left\{t\left(\begin{array}{cc}
\tilde{M} & -2 \tilde{Q}^{T} \tilde{Q} \\
\tilde{\Gamma} & -\tilde{M}^{T}
\end{array}\right)\right\}
$$

and $\tilde{M}, \tilde{Q}^{T} \tilde{Q}, \tilde{\Gamma}$ are given in Theorem 4.2.

However, the inverse $\psi_{22}^{-1}(t)$ does not necessarily exist and it is hard to give conditions on the parameters which imply this. The next proposition presents an alternative representation of the solution (under further parameter restrictions) which allows explicit conditions. As usual $\sinh (A)$ for $A \in S_{d}^{+}(\mathbb{R})$ is defined as $\sinh (A)=\frac{e^{A}-e^{-A}}{2}$ where $e^{A}$ is the matrix exponential. Moreover, the log which appears there is the matrix logarithm. The proof can be found in the appendix.

Proposition 4.4. Suppose $\tilde{M}^{T}\left(\tilde{Q}^{T} \tilde{Q}\right)^{-1}=\left(\tilde{Q}^{T} \tilde{Q}\right)^{-1} \tilde{M}$. For $t \in[0, T]$ define

$$
\begin{aligned}
\kappa(t) & :=-\left(\sqrt{C_{2}} \cosh \left(\sqrt{C_{2}} t\right)+C_{1} \sinh \left(\sqrt{C_{2}} t\right)\right)^{-1}\left(\sqrt{C_{2}} \sinh \left(\sqrt{C_{2}} t\right)+C_{1} \cosh \left(\sqrt{C_{2}} t\right),\right. \\
C_{2} & :=\tilde{Q}\left(-2 \tilde{\Gamma}+\tilde{M}^{T}\left(\tilde{Q}^{T} \tilde{Q}\right)^{-1} \tilde{M}\right) \tilde{Q}^{T} \in S_{d}(\mathbb{R}), \\
C_{1} & :=-\tilde{Q} \tilde{M}^{T}\left(\tilde{Q}^{T} \tilde{Q}\right)^{-1} \tilde{Q}^{T} \in S_{d}(\mathbb{R})
\end{aligned}
$$

with $\tilde{M}, \tilde{Q}^{T} \tilde{Q}, \tilde{\Gamma}$ given in Theorem 4.2. If

$$
-2 \tilde{\Gamma}+\tilde{M}^{T}\left(\tilde{Q}^{T} \tilde{Q}\right)^{-1} \tilde{M} \succ 0, \quad \sqrt{C_{2}}+C_{1} \succeq 0 \quad \text { and } \quad \tilde{Q}^{T} \tilde{Q} \in G L_{d}(\mathbb{R})
$$

is satisfied, then the partial differential equation (4.3) possesses on $[0, T]$ the finite solution (4.8), with

$$
\begin{aligned}
& \psi(t)=\frac{1}{2}\left(\tilde{Q}^{-1} \sqrt{C_{2}} \kappa(t) \tilde{Q}^{-T}\right)-\frac{1}{2} \tilde{M}^{T}\left(\tilde{Q}^{T} \tilde{Q}\right)^{-1} \\
& \phi(t)=-\frac{1}{2} \operatorname{Tr}\left(N N^{T} \tilde{M}^{T}\left(\tilde{Q}^{T} \tilde{Q}\right)^{-1}\right) t+\gamma r t \\
& -\frac{1}{2} \operatorname{Tr}\left(\tilde{Q}^{-T} N N^{T} \tilde{Q}^{-1} \cdot \log \left[\left(\sqrt{C_{2}}\right)^{-1}\left(\sqrt{C_{2}} \cosh \left(\sqrt{C_{2}} t\right)+C_{1} \sinh \left(\sqrt{C_{2}} t\right)\right)\right]\right) .
\end{aligned}
$$

Remark 4.5. $\quad$ a) Note that the condition $\tilde{M}^{T}\left(\tilde{Q}^{T} \tilde{Q}\right)^{-1}=\left(\tilde{Q}^{T} \tilde{Q}\right)^{-1} \tilde{M}$ is more general than the commutativity assumption $\tilde{M} \tilde{Q}=\tilde{Q} \tilde{M}$ in $\operatorname{Bru}$ (1991). For a discussion see Gnoatto \& Grasselli (2012).

b) The first two conditions in 4.11 for the existence of a finite solution of the Riccati equations reduce to the single condition

$$
\tilde{\Gamma} \prec \tilde{M}^{T}\left(2 \tilde{Q}^{T} \tilde{Q}\right)^{-1} \tilde{M},
$$

if $\tilde{M}^{T}\left(\tilde{Q}^{T} \tilde{Q}\right)^{-1}$ is negative semidefinite. Under this assumption, $C_{1}$ is positive semidefinite, thus the condition (4.14) implies the condition $\sqrt{C_{2}}+C_{1} \succ 0$. If $\gamma>0$ and $Q \in G L_{d}(\mathbb{R})$ then $\tilde{Q}^{T} \tilde{Q} \in G L_{d}(\mathbb{R})$ is always satisfied. Note that for $\gamma<0$ the condition $\tilde{\Gamma} \preceq \tilde{M}^{T}\left(2 \tilde{Q}^{T} \tilde{Q}\right)^{-1} \tilde{M}$ is always fulfilled. 
c) In the special case $d=1$ the conditions (4.11) coincide with the results in the Heston model in Proposition 5.2 of Kraft (2005). Let us use the terminologies in Kraft (2005) and denote for $d=1$

$$
M:=-\frac{\kappa}{2}<0, \quad \mathbf{v}:=\bar{\lambda}, \quad \rho:=\rho, \quad Q:=\frac{\sigma}{2}, \quad \tilde{M}=-\frac{\kappa}{2}+\frac{\gamma}{1-\gamma} \frac{\sigma}{2} \rho \bar{\lambda}=:-\frac{\tilde{\kappa}}{2} .
$$

In Kraft $(2005)$ it is assumed that $\tilde{\kappa}>0$. Then $\tilde{Q}^{T} \tilde{Q}=\frac{\sigma^{2}}{4}\left(1+\frac{\gamma}{1-\gamma} \rho^{2}\right)=: \tilde{\sigma}^{2}$ and $2 \tilde{M}^{T}\left(\tilde{Q}^{T} \tilde{Q}\right)^{-1}=-\frac{\tilde{\kappa}}{\tilde{\sigma}^{2}}$ is negative semidefinite. The condition in 4.14) can be written as

$$
\frac{\gamma}{2(1-\gamma)} \bar{\lambda}^{2}<\left(\kappa^{2}-\frac{2 \gamma \bar{\lambda} \rho \sigma \kappa}{(1-\gamma)}+\frac{\gamma^{2} \bar{\lambda}^{2} \rho^{2} \sigma^{2}}{(1-\gamma)^{2}}\right) \cdot \frac{1}{2\left(\sigma^{2}+\frac{\gamma}{(1-\gamma)} \rho^{2} \sigma^{2}\right)} .
$$

Note that the term $\sigma^{2}+\frac{\gamma}{(1-\gamma)} \rho^{2} \sigma^{2}$ is always positive, thus, multiplying both sides with this expression, the inequality above can be simplified to

$$
\frac{\gamma \bar{\lambda}}{1-\gamma}\left(\frac{\bar{\lambda}}{2}+\frac{\rho \kappa}{\sigma}\right)<\frac{\kappa^{2}}{2 \sigma^{2}}
$$

which is condition (26) in Kraft (2005).

d) The one-dimensional Heston model with power utility has also been solved in Kallsen \& Muhle-Karbe (2010) using martingale methods. They deal with parameter settings where the value function is finite for certain time horizons up to a critical one. The conditions in (4.11) ensure that the value function exists for all $T \geq 0$. Inspecting the proof of Proposition 4.4 one may also be able to identify cases where the matrix $\sqrt{C_{2}} \cosh \left(\sqrt{C_{2}} t\right)+C_{1} \sinh \left(\sqrt{C_{2}} t\right) \in G L_{d}(\mathbb{R})$ for some $t$ up to a critical one.

\section{VERIFICATION}

In this section we consider the linear drift case from Section 4.2 and verify that

$$
G(t, x, \Sigma):=\frac{x^{\gamma}}{\gamma} \exp (\phi(T-t)+\operatorname{Tr}[\psi(T-t) \Sigma]), \quad t \in[0, T]
$$

where $\psi, \phi$ are solutions of $(4.9),(4.10)$ is indeed the value function of our portfolio optimization problem, given $G(t, x, \Sigma)$ is finite which is for example satisfied under the conditions of Proposition 4.4 .

First note that our candidate for the optimal portfolio strategy $\pi^{*}$ is given through (3.3) by $\pi_{t}^{*}=\pi^{*}\left(t, \Sigma_{t}\right)$ with

$$
\pi^{*}(t, \Sigma)=\frac{\mathbf{v}}{1-\gamma}+\frac{2 \psi(T-t) Q^{T} \boldsymbol{\rho}}{1-\gamma}
$$

Note that $\pi^{*}(t, \Sigma)=\pi_{t}^{*}$, i.e. the optimal strategy is purely deterministic and does not depend on $\Sigma$.

We will show directly that the corresponding value attained by this portfolio strategy is $G(t, x, \Sigma)$ and every other admissible portfolio strategy will not yield a larger value.

Theorem 5.1 (Verification). Suppose the function $G$ in (5.1) is finite (this is for example satisfied under the conditions of Proposition 4.4). Given $\left(\pi_{t}^{*}\right)$ as in (5.2), there is

$$
\mathbb{E}^{t, x, \Sigma}\left[\frac{\left(X_{T}^{\pi^{*}}\right)^{\gamma}}{\gamma}\right]=G(t, x, \Sigma), \quad t \in[0, T], x>0, \Sigma \in S_{d}^{+}(\mathbb{R})
$$

and for every other admissible portfolio strategy $\pi$ we obtain

$$
\mathbb{E}^{t, x, \Sigma}\left[\frac{\left(X_{T}^{\pi}\right)^{\gamma}}{\gamma}\right] \leq G(t, x, \Sigma), \quad t \in[0, T], x>0, \Sigma \in S_{d}^{+}(\mathbb{R}) .
$$

Thus, $V=G$ and $\left(\pi_{t}^{*}\right)$ in 5.2 is the optimal portfolio strategy. 
Proof. The inequality for every admissible portfolio strategy is standard and follows e.g. like in Kraft (2005) Proposition 4.3. For the equation recall that we have

$$
X_{T}^{\pi}=X_{t}^{\pi} \exp \left(\int_{t}^{T}\left[\pi_{s}^{T} \Sigma_{s} \mathbf{v}+r-\frac{1}{2}\left\|\pi_{s}^{T} \Sigma_{s}^{1 / 2}\right\|_{2}^{2}\right] d s+\int_{t}^{T} \pi_{s}^{T} \Sigma_{s}^{1 / 2} d W_{s}^{S}\right)
$$

with $X_{t}^{\pi}=x$. Let us denote

$$
Z_{t}:=\left.\frac{d \mathbb{Q}}{d \mathbb{P}}\right|_{\mathcal{F}_{t}}=\exp \left(\gamma \int_{t}^{T}\left(\pi_{s}^{*}\right)^{T} \Sigma_{s}^{1 / 2} d W_{s}^{S}-\frac{\gamma^{2}}{2} \int_{t}^{T}\left\|\left(\pi_{s}^{*}\right)^{T} \Sigma_{s}^{1 / 2}\right\|^{2} d s\right),
$$

which is a martingale by Proposition 8.2 in the appendix. Using Girsanov's Theorem we obtain

$$
\begin{aligned}
& x^{-\gamma} \mathbb{E}^{t, x, \Sigma}\left[\left(X_{T}^{\pi^{*}}\right)^{\gamma}\right] \\
= & \mathbb{E}^{t, x, \Sigma}\left[\exp \left(\gamma \int_{t}^{T}\left[\left(\pi_{s}^{*}\right)^{T} \Sigma_{s} \mathbf{v}+r-\frac{1}{2}\left\|\left(\pi_{s}^{*}\right)^{T} \Sigma_{s}^{1 / 2}\right\|_{2}^{2}\right] d s+\gamma \int_{t}^{T}\left(\pi_{s}^{*}\right)^{T} \Sigma_{s}^{1 / 2} d W_{s}^{S}\right)\right] \\
= & \mathbb{E}_{\mathbb{Q}}^{t, x, \Sigma}\left[\exp \left(\gamma \int_{t}^{T}\left[\left(\pi_{s}^{*}\right)^{T} \Sigma_{s} \mathbf{v}+r-\frac{1}{2}\left\|\left(\pi_{s}^{*}\right)^{T}\left(\Sigma_{s}\right)^{1 / 2}\right\|_{2}^{2}+\frac{\gamma}{2}\left\|\left(\pi_{s}^{*}\right)^{T}\left(\Sigma_{s}\right)^{1 / 2}\right\|_{2}^{2}\right] d s\right)\right] \\
= & \mathbb{E}_{\mathbb{Q}}^{t, x, \Sigma}\left[\exp \left(\gamma \int_{t}^{T}\left[\left(\pi_{s}^{*}\right)^{T} \Sigma_{s} \mathbf{v}+r+\frac{\gamma-1}{2}\left(\pi_{s}^{*}\right)^{T} \Sigma_{s} \pi_{s}^{*}\right] d s\right)\right] \\
= & \mathbb{E}_{\mathbb{Q}}^{t, x, \Sigma}\left[\exp \left(\gamma r(T-t)+\int_{t}^{T} \operatorname{Tr}\left[\left(\gamma \mathbf{v}\left(\pi_{s}^{*}\right)^{T}+\frac{\gamma(\gamma-1)}{2} \pi_{s}^{*}\left(\pi_{s}^{*}\right)^{T}\right) \Sigma_{s}\right] d s\right)\right] .
\end{aligned}
$$

In what follows let us introduce the deterministic matrix-valued process

$$
F_{s}=\gamma \mathbf{v}\left(\pi_{s}^{*}\right)^{T}+\frac{\gamma(\gamma-1)}{2} \pi_{s}^{*}\left(\pi_{s}^{*}\right)^{T}
$$

which appears in (5.4). By plugging in the optimal strategy $\pi^{*}$ and using 4.9 we obtain:

$$
\begin{aligned}
F_{s}= & \frac{\gamma}{1-\gamma}\left\{\frac{1}{2} \mathbf{v} \mathbf{v}^{T}+\mathbf{v} \boldsymbol{\rho}^{T} Q \psi(T-s)-\psi(T-s) Q^{T} \boldsymbol{\rho} \mathbf{v}^{T}-2 \psi(T-s) Q^{T} \boldsymbol{\rho} \boldsymbol{\rho}^{T} Q \psi(T-s)\right\} \\
= & \psi_{t}(T-s)-\psi(T-s) M-M^{T} \psi(T-s)-2 \psi(T-s) Q^{T} Q \psi(T-s) \\
& +\frac{\gamma}{\gamma-1}\left\{2 \psi(T-s) Q^{T} \boldsymbol{\rho} \mathbf{v}^{T}+4 \psi(T-s) Q^{T} \boldsymbol{\rho} \boldsymbol{\rho}^{T} Q \psi(T-s)\right\} .
\end{aligned}
$$

Now note that under $\mathbb{Q}$ defined in $(5.3)$ the process $\hat{W}_{t}^{\sigma}:=W_{t}^{\sigma}-\gamma \Sigma_{t}^{1 / 2} \pi_{t}^{*} \boldsymbol{\rho}^{T}$ is also a standard Brownian motion and the dynamics of $\left(\Sigma_{t}\right)$ under $\mathbb{Q}$ is given by

$$
\begin{aligned}
d \Sigma_{t} & =\left(N N^{T}+M \Sigma_{t}+\Sigma_{t} M^{T}\right) d t+\Sigma_{t}^{1 / 2} d W_{t}^{\sigma} Q+Q^{T}\left(d W_{t}^{\sigma}\right)^{T} \Sigma_{t}^{1 / 2} \\
& =\left(N N^{T}+M \Sigma_{t}+\Sigma_{t} M^{T}+\gamma \Sigma_{t} \pi^{*} \rho^{T} Q+\gamma Q^{T} \boldsymbol{\rho}\left(\pi^{*}\right)^{T} \Sigma_{t}\right) d t+\Sigma_{t}^{1 / 2} d \hat{W}_{t}^{\sigma} Q+Q^{T}\left(d \hat{W}_{t}^{\sigma}\right)^{T} \Sigma_{t}^{1 / 2} .
\end{aligned}
$$

Hence under $\mathbb{Q}$ the process $\left(\Sigma_{t}\right)$ is again a Wishart process with drift

$$
\begin{aligned}
& N N^{T}+M \Sigma_{t}+\Sigma_{t} M^{T}+\gamma \Sigma_{t} \pi^{*} \boldsymbol{\rho}^{T} Q+\gamma Q^{T} \boldsymbol{\rho}\left(\pi^{*}\right)^{T} \Sigma_{t} \\
= & N N^{T}+M \Sigma_{t}+\Sigma_{t} M^{T}+\frac{\gamma}{1-\gamma} \Sigma_{t} \mathbf{v} \boldsymbol{\rho}^{T} Q+\frac{2 \gamma}{1-\gamma} \Sigma_{t} \psi(T-t) Q^{T} \boldsymbol{\rho} \boldsymbol{\rho}^{T} Q \\
& +\frac{\gamma}{1-\gamma} Q^{T} \boldsymbol{\rho} \mathbf{v}^{T} \Sigma_{t}+\frac{2 \gamma}{1-\gamma} Q^{T} \boldsymbol{\rho} \boldsymbol{\rho}^{T} Q \psi(T-t) \Sigma_{t} .
\end{aligned}
$$

Next we compute $\int_{t}^{T} \operatorname{Tr}\left(F_{s} \Sigma_{s}\right) d s$ under $\mathbb{Q}$. For this instance note that due to the product rule and since $\psi(0)=\mathbf{0}, \Sigma_{t}=\Sigma$ we obtain

$$
\int_{t}^{T} \operatorname{Tr}\left(\psi_{t}(T-s) \Sigma_{s}\right) d s=\operatorname{Tr}\left(\Sigma \psi(T-t)+\int_{t}^{T} \psi(T-s) d \Sigma_{s}\right) .
$$


Plugging in the dynamics of $\left(\Sigma_{t}\right)$ under $\mathbb{Q}$ we obtain:

$$
\begin{aligned}
& \int_{t}^{T} \operatorname{Tr}\left(F_{s} \Sigma_{s}\right) d s \\
= & \operatorname{Tr}(\Sigma \psi(T-t))+\int_{t}^{T} \operatorname{Tr}\left(\psi(T-s) N N^{T}\right) d s-\int_{t}^{T} \operatorname{Tr}\left(2 \psi(T-s) Q^{T} Q \psi(T-s) \Sigma_{s}\right) d s \\
& +\operatorname{Tr}\left(\int_{t}^{T} \psi(T-s) \Sigma_{s}^{1 / 2} d \hat{W}_{s}^{\sigma} Q+\int_{t}^{T} \psi(T-s) Q^{T}\left(d \hat{W}_{s}^{\sigma}\right)^{T} \Sigma_{s}^{1 / 2}\right) .
\end{aligned}
$$

Note that the differential equation 4.10 can be written as

$$
\phi(T-t)=\int_{t}^{T} \operatorname{Tr}\left(\psi(T-s) N N^{T}\right) d s+\gamma r(T-t) .
$$

Hence we obtain:

$$
\begin{aligned}
& x^{-\gamma} \mathbb{E}^{t, x, \Sigma}\left[\left(X_{T}^{\pi^{*}}\right)^{\gamma}\right] \\
= & \mathbb{E}_{\mathbb{Q}}^{t, x, \Sigma}\left[\exp \left(\gamma r(T-t)+\int_{t}^{T} \operatorname{Tr}\left(F_{s} \Sigma_{s}\right) d s\right)\right] \\
= & \mathbb{E}_{\mathbb{Q}}^{t, x, \Sigma}\left[\operatorname { e x p } \left(\operatorname{Tr}(\Sigma \psi(T-t))+\phi(T-t)-\int_{t}^{T} \operatorname{Tr}\left(2 \psi(T-s) Q^{T} Q \psi(T-s) \Sigma_{s}\right) d s\right.\right. \\
& \left.\left.+\operatorname{Tr}\left(\int_{t}^{T} \psi(T-s) \Sigma_{s}^{1 / 2} d \hat{W}_{s}^{\sigma} Q+\int_{t}^{T} \psi(T-s) Q^{T}\left(d \hat{W}_{s}^{\sigma}\right)^{T} \Sigma_{s}^{1 / 2}\right)\right)\right] \\
= & \exp (\operatorname{Tr}(\Sigma \psi(T-t))+\phi(T-t)) \\
& \mathbb{E}_{\mathbb{Q}}^{t, x, \Sigma}\left[\exp \left(-\int_{t}^{T} \operatorname{Tr}\left(2 Q \psi(T-s) \Sigma_{s} \psi(T-s) Q^{T}\right) d s+2 \operatorname{Tr}\left(\int_{t}^{T} Q \psi(T-s) \Sigma_{s}^{1 / 2} d \hat{W}_{s}^{\sigma}\right)\right)\right] .
\end{aligned}
$$

With the help of Proposition 8.1 it can be shown that the expression within the expectation is a $\mathbb{Q}$-martingale with expectation 1 which yields:

$$
\mathbb{E}^{t, x, \Sigma}\left[\frac{\left(X_{T}^{\pi^{*}}\right)^{\gamma}}{\gamma}\right]=\frac{x^{\gamma}}{\gamma} \exp (\operatorname{Tr}(\Sigma \psi(T-t))+\phi(T-t))
$$

and the statement is shown.

Remark 5.2. The optimal portfolio strategy $\left(\pi_{t}^{*}\right)$ in 5.2 can be decomposed into the Merton ratio $\frac{\mathbf{v}}{1-\gamma}$ and the hedging demand given by

$$
\frac{2 \psi(T-t) Q^{T} \boldsymbol{\rho}}{1-\gamma}
$$

In case there is no correlation between the Brownian motions that drive the assets and those which drive the volatility process, i.e. $\rho=0$, the optimal portfolio strategy reduces to the Merton ratio and does not depend on time. In any case note that the optimal portfolio strategy does not depend on $N N^{T}$ and is deterministic.

\section{Logarithmic Utility Case}

In case the utility function in problem (2.6) is the logarithmic utility $U(x)=\log x$, the problem can be solved by pointwise maximization. Indeed we obtain in the general drift case 
for an admissible portfolio strategy:

$$
\begin{aligned}
& \mathbb{E}^{t, x, \Sigma}\left(\log \left(X_{T}^{\pi}\right)\right) \\
= & \log x+\mathbb{E}^{t, x, \Sigma}\left[\int_{t}^{T} \pi_{s}^{T}\left(B\left(\Sigma_{s}\right)-\mathbf{r}\right)+r-\frac{1}{2}\left\|\pi_{s}^{T} \Sigma_{s}^{1 / 2}\right\|_{2}^{2} d s\right]+\mathbb{E}^{t, x, \Sigma}\left[\int_{t}^{T} \pi_{s}^{T} \Sigma_{s}^{1 / 2} d W_{s}^{S}\right] \\
= & \log x+\mathbb{E}^{t, x, \Sigma}\left[\int_{t}^{T} \max _{\pi_{s}, s \in[t, T]}\left(\pi_{s}^{T}\left(B\left(\Sigma_{s}\right)-\mathbf{r}\right)+r-\frac{1}{2}\left\|\pi_{s}^{T} \Sigma_{s}^{1 / 2}\right\|_{2}^{2}\right) d s\right]
\end{aligned}
$$

when we assume that $\int_{t}^{T} \pi_{s}^{T} \Sigma_{s}^{1 / 2} d W_{s}^{S}$ is a true martingale. Obviously the maximizer is here given by

$$
\pi^{*}(\Sigma)=\Sigma^{-1}(B(\Sigma)-\mathbf{r}) .
$$

For the case $B(\Sigma)=r+\Sigma \mathbf{v}$ with a $\mathbf{v} \in \mathbb{R}^{d}$, it is evident that $\pi_{t}^{*} \equiv \mathbf{v}$ and $\int_{t}^{T} \mathbf{v}^{T} \Sigma_{s}^{1 / 2} d W_{s}^{S}$ is a true martingale.

Plugging $\pi^{*}$ into 6.2 yields

$$
\sup _{\left(\pi_{s}\right)} \mathbb{E}^{t, x, \Sigma}\left(\log \left(X_{T}^{\pi}\right)\right)=\log x+r(T-t)+\frac{1}{2} \int_{t}^{T} E^{t, x, \Sigma}\left(\left(B\left(\Sigma_{s}\right)-\mathbf{r}\right)^{T} \Sigma_{s}^{-1}\left(B\left(\Sigma_{s}\right)-\mathbf{r}\right)\right) d s .
$$

For further computations we need to calculate the conditional expectation of the function

$$
f\left(\Sigma_{t}\right):=\left(B\left(\Sigma_{t}\right)-\mathbf{r}\right)^{T} \Sigma_{t}^{-1}\left(B\left(\Sigma_{t}\right)-\mathbf{r}\right) .
$$

Note that $\Sigma_{t}$ has a Wishart distribution for fixed $t$. We refer to Matsumoto (2012) for the conditional expectation of the moments of real inverse Wishart distributed matrices and Sultan \& Tracy (1996) for the moments of central and noncentral Wishart distributions.

\section{An Example}

In this subsection we compute the optimal portfolio strategy numerically for time horizon $T=1$ in a two-dimensional example for the case $B(\Sigma)-\mathbf{r}=\Sigma \mathbf{v}$. More precisely, we consider a financial market with one riskfree asset and $d=2$ risky assets. The parameters are taken from Buraschi et al. (2010) who calibrated such a model to real market data of the S\& P500 Index and 30-year Treasury bonds. For the volatility process $\left(\Sigma_{t}\right)$ they obtain

$$
M=\left(\begin{array}{cc}
-1.21 & 0.491 \\
0.3292 & -1.271
\end{array}\right), Q=\left(\begin{array}{cc}
0.167 & 0.033 \\
0.001 & 0.09
\end{array}\right), \boldsymbol{\rho}=\left(\begin{array}{l}
-0.115 \\
-0.549
\end{array}\right), \mathbf{v}=\left(\begin{array}{l}
4.722 \\
3.317
\end{array}\right) .
$$

In the computation we assume an investor with power utility $U(x)=\frac{1}{\gamma} x^{\gamma}, \gamma<1, \gamma \neq 0$ where we vary the parameter $\gamma$. We use the formula in Proposition 4.3 to compute $\psi(t)$, i.e.

$$
\psi(t)=\psi_{22}(t)^{-1} \psi_{21}(t), \quad t \in[0,1]
$$

where

$$
\left(\begin{array}{ll}
\psi_{11}(t) & \psi_{12}(t) \\
\psi_{21}(t) & \psi_{22}(t)
\end{array}\right)=\exp \left\{t\left(\begin{array}{cc}
\tilde{M} & -2 \tilde{Q}^{T} \tilde{Q} \\
\tilde{\Gamma} & -\tilde{M}^{T}
\end{array}\right)\right\}, \quad t \in[0,1]
$$

and $\tilde{M}, \tilde{Q}^{T} \tilde{Q}, \tilde{\Gamma}$ are given in Theorem 4.2 . In our numerical study, $\psi_{22}(t)$ was always invertible and we obtained finite values for $\psi(t)$. The optimal portfolio strategy is given by (5.2):

$$
\pi_{t}^{*}=\frac{\mathbf{v}}{1-\gamma}+\frac{2 \psi(T-t) Q^{T} \boldsymbol{\rho}}{1-\gamma} .
$$

Indeed, in the figures we only plot the hedging demand.

Note that the degree of risk aversion of the investor changes with parameter $\gamma$. Formally the degree of risk aversion is defined by the Arrow-Pratt absolute risk aversion coefficient, which is

$$
-\frac{U^{\prime \prime}(x)}{U^{\prime}(x)}=(1-\gamma) \frac{1}{x}
$$



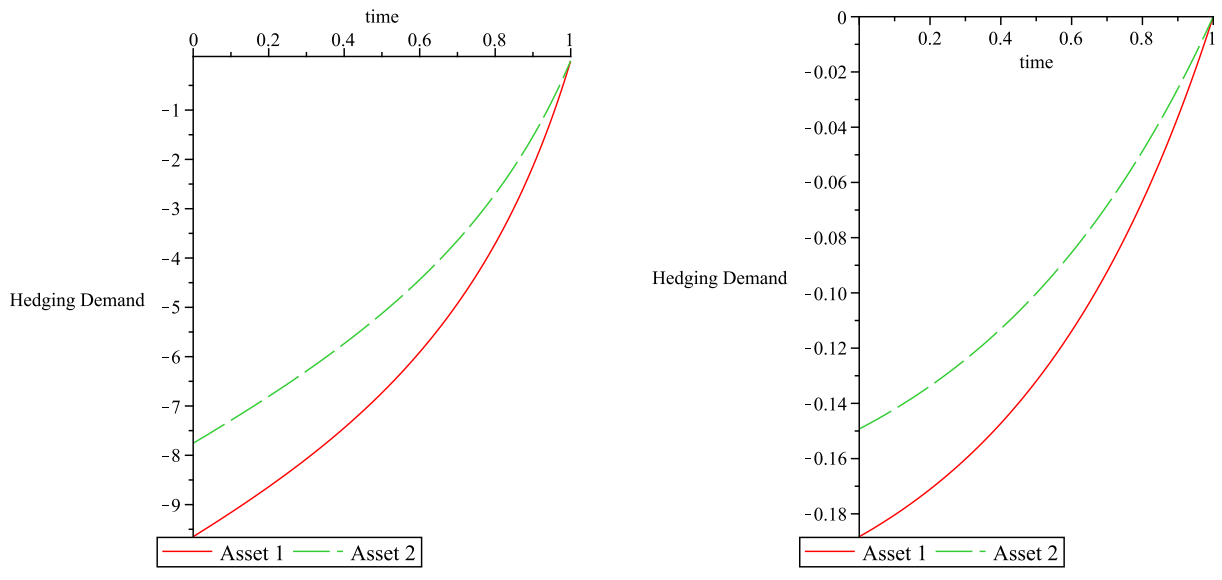

Figure 1. Hedging demand $\frac{2}{1-\gamma} \psi(1-t) Q^{T} \boldsymbol{\rho}$ for asset 1 and 2 for (from left to right) $\gamma=0.8 \gamma=0.2$.
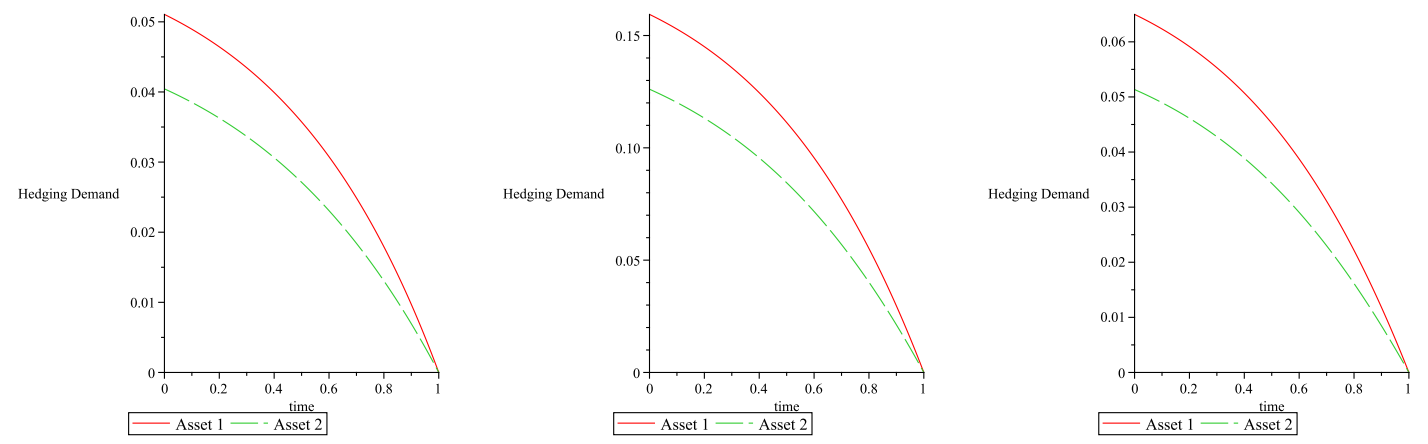

FIGURE 2. Hedging demand $\frac{2}{1-\gamma} \psi(1-t) Q^{T} \boldsymbol{\rho}$ for asset 1 and 2 for (from left to right) $\gamma=-0.1, \gamma=-1, \gamma=-8$.

in case of the power utility. Thus, the risk aversion decreases for all wealth levels with $\gamma$. The case $\gamma \rightarrow 0$ corresponds to the logarithmic utility: It is easy to see that $\gamma \rightarrow 0$ implies $\tilde{\Gamma} \rightarrow 0$ and hence a trivial solution of 4.9 is $\psi \equiv 0$. Thus, the hedging demand

$$
\frac{2 \psi(T-t) Q^{T} \boldsymbol{\rho}}{1-\gamma}
$$

in this case is zero and $\pi_{t}^{\star}=\mathbf{v}$. We know from 6.3 that this is the optimal portfolio strategy in case of a logarithmic utility.

If $\gamma \in(0,1)$, the investor is less risk averse than in the logarithmic utility case and we expect a negative hedging demand which implies a tendency to shortsell stocks. Indeed, when we look at 
the pictures of the hedging demand for different $\gamma$ we see that positive $\gamma$ (less risk averse investor) imply a negative hedging demand (see figure 1) and negative $\gamma$ (more risk averse investor) imply a positive hedging demand (see figure 2). We have observed this behavior in all our numerical computations. This effect has also been reported in other situations with power utility (see e.g. Rieder \& Bäuerle (2005)). Since the formula for $\left(\pi_{t}^{*}\right)$ is still quite complicated we did not try to prove this observation but we conjecture that it is true for reasonable parameters.

In any case, the numerical study shows that it is very easy to compute the optimal portfolio strategy numerically since the formulas are explicit and no numerical integration is needed. Much higher dimensions than $d=2$ would also pose no numerical problems.

\section{Appendix}

This section contains some proofs and auxiliary results.

Proof of Theorem 4.1: Observe first that under the new measure $\tilde{\mathbb{P}}$, the process defined by $\tilde{W}_{t}^{\sigma}:=W_{t}^{\sigma}-\int_{0}^{t} \theta\left(\Sigma_{s}\right) d s$ is a $d \times d$ matrix Brownian motion on $[0, T]$ by the Girsanov theorem. Thus, under $\tilde{\mathbb{P}}$ the process $\left(\Sigma_{t}\right)$ has dynamics

$$
d \Sigma_{t}=\left(N N^{T}+M \Sigma_{t}+\Sigma_{t} M^{T}+H\right) d t+\Sigma_{t}^{1 / 2} d \tilde{W}_{t}^{\sigma} Q+Q^{T}\left(d \tilde{W}_{t}^{\sigma}\right)^{T} \Sigma_{t}^{1 / 2}
$$

where we have used that $\Sigma^{1 / 2} \theta Q+Q^{T} \theta^{T} \Sigma^{1 / 2}=H$. Hence the process $\left(\Sigma_{t}\right)$ is again a Wishart process under $\tilde{\mathbb{P}}$ however with different drift. The characteristic operator of this process is for $f \in C^{2}\left(S_{d}^{+}(\mathbb{R})\right)$ given by

$$
(\mathcal{A} f)(\Sigma)=\frac{1}{2} \operatorname{Tr}\left(\Sigma\left(\nabla+\nabla^{T}\right)\left(Q^{T} Q\right)\left(\nabla^{T}+\nabla\right)\right) f+\operatorname{Tr}\left(\left(N N^{T}+M \Sigma+\Sigma M^{T}+H\right) \nabla f\right)
$$

under $\tilde{\mathbb{P}}$. Then the partial differential equation 4.3 can be written as

$$
\begin{aligned}
& \mathcal{A} h=-h_{t}-\left(\frac{\gamma r}{\delta}+\frac{\gamma}{2(1-\gamma) \delta}(B-\mathbf{r})^{T} \Sigma^{-1}(B-\mathbf{r})\right) h, \\
& h(T, \Sigma)=1 .
\end{aligned}
$$

Applying the theorem of Feynman-Kac, we conclude that the representation in (4.5) is the solution of 4.3) under proper conditions.

Proof of Theorem 4.2: We simply verify that $g$ given in (4.8) satisfies (4.7). To this end note that

$$
\begin{aligned}
g_{t} & =-g\left(\phi_{t}(T-t)+\operatorname{Tr}\left(\psi_{t}(T-t) \Sigma\right)\right), \\
\nabla g & =\nabla^{T} g=g \psi(T-t), \\
g_{\Sigma_{l k}, \Sigma_{i j}} & =g \psi_{j i}(T-t) \psi_{k l}(T-t) .
\end{aligned}
$$

Plugging these derivatives into (4.7) yields (obviously $g$ cancels out):

$$
\begin{aligned}
0= & -\left(\phi_{t}+\operatorname{Tr}\left(\psi_{t} \Sigma\right)\right)+2 \operatorname{Tr}\left(\Sigma \psi\left(Q^{T} Q\right) \psi\right) \\
& +\operatorname{Tr}\left(\psi\left(N N^{T}+M \Sigma+\Sigma M^{T}\right)\right)+\gamma r+\frac{\gamma}{2(1-\gamma)} \operatorname{Tr}\left(\mathbf{v}^{T} \Sigma \mathbf{v}+4 \boldsymbol{\rho}^{T} Q \psi \Sigma \mathbf{v}+4 \boldsymbol{\rho}^{T} Q \psi \Sigma \psi Q^{T} \boldsymbol{\rho}\right) .
\end{aligned}
$$


In order to see that the right hand side is really zero, keep in mind that $\phi$ satisfies 4.10 and $\psi$ satisfies 4.9 and that the following relations hold

$$
\begin{aligned}
& \frac{\gamma}{2(1-\gamma)} \operatorname{Tr}\left(\mathbf{v}^{T} \Sigma \mathbf{v}\right)=\operatorname{Tr}(\tilde{\Gamma} \Sigma), \\
& \frac{\gamma}{2(1-\gamma)} \operatorname{Tr}\left(4 \boldsymbol{\rho}^{T} Q \psi \Sigma \mathbf{v}\right)=\frac{\gamma}{(1-\gamma)} \operatorname{Tr}\left(\mathbf{v} \boldsymbol{\rho}^{T} Q \psi \Sigma\right)+\frac{\gamma}{(1-\gamma)} \operatorname{Tr}\left(\psi Q^{T} \boldsymbol{\rho} \mathbf{v}^{T} \Sigma\right), \\
& \operatorname{Tr}\left(\boldsymbol{\rho}^{T} Q \psi \Sigma \psi Q^{T} \boldsymbol{\rho}\right)=\operatorname{Tr}\left(\psi Q^{T} \boldsymbol{\rho} \boldsymbol{\rho}^{T} Q \psi \Sigma\right) .
\end{aligned}
$$

To ensure $g(T, \Sigma)=1$, we need the initial conditions $\psi(0)=\mathbf{0}$ and $\phi(0)=0$.

Proof of Proposition 4.4: The explicit representation of $\psi$ and $\phi$ follows directly from Theorem 11 in Gnoatto \& Grasselli (2012). Given $\tilde{Q}^{T} \tilde{Q} \in G L_{d}(\mathbb{R})$ these expressions are well-defined and finite when

$$
K(t):=\sqrt{C_{2}} \cosh \left(\sqrt{C_{2}} t\right)+C_{1} \sinh \left(\sqrt{C_{2}} t\right) \in G L_{d}(\mathbb{R}), \quad t \in[0, T] .
$$

Indeed we show now that $K(t) \in G L_{d}(\mathbb{R})$, for all $t \geq 0$, if and only if

$$
-2 \tilde{\Gamma}+\tilde{M}^{T}\left(\tilde{Q}^{T} \tilde{Q}\right)^{-1} \tilde{M} \succ 0 \quad \text { and } \quad \sqrt{C_{2}}+C_{1} \succeq 0 .
$$

First of all note that $C_{2}$ is symmetric and for the well definedness of the matrix square root of $C_{2}$, we need $C_{2}$ to be nonnegative definite, which is equivalent to $-2 \tilde{\Gamma}+\tilde{M}^{T}\left(\tilde{Q}^{T} \tilde{Q}\right)^{-1} \tilde{M} \succeq 0$. Consider the situation $t=0$. We obtain $K(0)=\sqrt{C_{2}}$. Then $K(0) \in G L_{d}(\mathbb{R})$ if and only if $-2 \tilde{\Gamma}+\tilde{M}^{T}\left(\tilde{Q}^{T} \tilde{Q}\right)^{-1} \tilde{M} \succ 0$.

Subsequently, we show the sufficiency of the conditions for $t>0$. For the sake of simplicity, we write

$$
K(t)=\frac{1}{2}\left(\sqrt{C_{2}}+C_{1}\right) e^{\sqrt{C_{2}} t}+\frac{1}{2}\left(\sqrt{C_{2}}-C_{1}\right) e^{-\sqrt{C_{2}} t} .
$$

Note that for $\sqrt{C_{2}} \in S_{d}^{+}(\mathbb{R})$ and $t>0$, there is $e^{\sqrt{C_{2}} t} \in S_{d}^{+}(\mathbb{R})$ and $e^{-\sqrt{C_{2}} t}=\left(e^{\sqrt{C_{2}} t}\right)^{-1} \in S_{d}^{+}(\mathbb{R})$. Moreover, expanding the matrix exponential functions as a series, one gets $e^{\sqrt{C_{2}} t}-e^{-\sqrt{C_{2}} t} \succ 0$ for $\sqrt{C_{2}} \in S_{d}^{+}(\mathbb{R}), t>0$. Hence, one can write $e^{\sqrt{C_{2}} t}=e^{-\sqrt{C_{2}} t}+P(t)$ with $P(t) \in S_{d}^{+}(\mathbb{R})$. Then there is

$$
\begin{aligned}
K(t) & =\frac{1}{2}\left(\sqrt{C_{2}}+C_{1}\right)\left(e^{-\sqrt{C_{2}} t}+P(t)\right)+\frac{1}{2}\left(\sqrt{C_{2}}-C_{1}\right) e^{-\sqrt{C_{2}} t} \\
& =\sqrt{C_{2}} e^{-\sqrt{C_{2}} t}+\frac{1}{2}\left(\sqrt{C_{2}}+C_{1}\right) P(t),
\end{aligned}
$$

which is always invertible if and only if $\operatorname{det}(K(t)) \neq 0$, for all $t>0$. Note that $\operatorname{det}\left(K(t) P^{-1}(t)\right)=$ $\operatorname{det}(K(t)) \operatorname{det}\left(P^{-1}(t)\right)$ and $\operatorname{det}\left(P^{-1}(t)\right)>0$, for all $t>0$, since $P(t) \in S_{d}^{+}(\mathbb{R})$ for all positive $t$, one gets $K(t) \in G L_{d}$ if and only if $\operatorname{det}\left(K(t) P^{-1}(t)\right) \neq 0$, for all $t>0$. Because of

$$
\begin{aligned}
& K(t) P^{-1}(t)=\sqrt{C_{2}} e^{-\sqrt{C_{2}} t}\left(e^{\sqrt{C_{2}} t}-e^{-\sqrt{C_{2}} t}\right)^{-1}+\frac{1}{2}\left(\sqrt{C_{2}}+C_{1}\right) \\
& =\left(e^{2 \sqrt{C_{2}} t}\left(\sqrt{C_{2}}\right)^{-1}-\left(\sqrt{C_{2}}\right)^{-1}\right)^{-1}+\frac{1}{2}\left(\sqrt{C_{2}}+C_{1}\right) \\
& =\underbrace{\left(2 t I+2 t^{2} \sqrt{C_{2}}+\frac{4 t^{3}}{3} C_{2}+\ldots\right)^{-1}}_{\succ 0}+\frac{1}{2}\left(\sqrt{C_{2}}+C_{1}\right) \text {, }
\end{aligned}
$$

it follows $\operatorname{det}\left(K(t) P^{-1}(t)\right) \neq 0$, for all $t>0$ from $\sqrt{C_{2}}+C_{1} \succeq 0$.

Eventually, we show the necessity of the conditions. We assume $\sqrt{C_{2}}+C_{1} \nsucceq 0$. Note that for $t=0, K(0)=\sqrt{C_{2}} \succ 0$, which implies that all the eigenvalues of $K(0)$ are positive. If $\sqrt{C_{2}}+C_{1} \nsucceq 0$, i.e. $\sqrt{C_{2}}+C_{1}$ possesses at least one negative eigenvalue, one can identify the 
matrix $\left(\sqrt{C_{2}}+C_{1}\right) e^{\sqrt{C_{2}} t}$ owns also at least one negative eigenvalue through a matrix similarity transformation:

$$
\sigma\left(\left(\sqrt{C_{2}}+C_{1}\right) e^{\sqrt{C_{2}} t}\right)=\sigma\left(\left(e^{\sqrt{C_{2}} t}\right)^{1 / 2}\left(\sqrt{C_{2}}+C_{1}\right)\left(e^{\sqrt{C_{2}} t}\right)^{1 / 2}\right),
$$

where $\sigma(A)$ denotes the spectrum of the matrix $A$. Then for $t$ large enough, it follows that $K(t)$ owns at least one negative eigenvalue. Since the spectrum of a matrix is a continuous function on the entries of the matrix (see Rellich (1969)), we conclude that there exists a $t>0$ with $K(t) \notin G L_{d}(\mathbb{R})$, if $\sqrt{C_{2}}+C_{1} \nsucceq 0$.

Proposition 8.1. Let us denote

$$
Z_{t}:=\exp \left(\int_{t}^{T} \operatorname{Tr}\left(A_{s} \Sigma_{s}^{1 / 2} d W_{s}^{\sigma}\right)-\frac{1}{2} \int_{t}^{T}\left\|A_{s} \Sigma_{s}^{1 / 2}\right\|^{2} d s\right),
$$

where $\left(A_{t}\right)_{t \in[0, T]}$ is a deterministic process with values in $\mathbb{R}^{d \times d}$ and bounded by $A^{*} \in \mathbb{R}^{d \times d}$. Then $\left(Z_{t}\right)_{t \in[0, T]}$ is a martingale.

Proof. By Lemma 4.2. in Hata \& Sekine (2011) we get that $\left(Z_{t}\right)$ is a martingale, if there is constant $C^{0} \in \mathbb{R}_{+}$s.t.

$$
\sqrt{\operatorname{Tr}\left(\theta(\Sigma) \theta^{T}(\Sigma) \theta(\Sigma) \theta^{T}(\Sigma)\right)} \leq C^{0} \sqrt{\operatorname{Tr}(\Sigma \Sigma)}
$$

with $\theta(\Sigma)=A \Sigma^{1 / 2}$. Consider the left-hand side of this inequality:

$$
\begin{aligned}
& \operatorname{Tr}\left(\theta(\Sigma) \theta^{T}(\Sigma) \theta(\Sigma) \theta^{T}(\Sigma)\right) \\
= & \operatorname{Tr}\left(A \Sigma A^{T} A \Sigma A^{T}\right) \leq \lambda_{\max } \operatorname{Tr}\left(A \Sigma \Sigma A^{T}\right)=\lambda_{\max } \operatorname{Tr}\left(\Sigma A^{T} A \Sigma\right) \leq \lambda_{\max }^{2} \operatorname{Tr}(\Sigma \Sigma),
\end{aligned}
$$

where $\lambda_{\max }$ is the largest eigenvalue of $A^{T} A$. The second last inequality follows from the fact that the trace of a matrix is the sum of its eigenvalues and

$$
A \Sigma A^{T} A \Sigma A^{T}=A \Sigma O \Lambda O^{T} \Sigma A^{T} \preceq \lambda_{\max } A \Sigma \Sigma A^{T},
$$

where $O \Lambda O^{T}$ is the spectral decomposition of $A^{T} A$. The last inequality follows in the same way, i.e.

$$
\Sigma A^{T} A \Sigma=\Sigma O \Lambda O^{T} \Sigma \preceq \lambda_{\max } \Sigma \Sigma .
$$

Since $A_{s} \in \mathbb{R}^{d \times d}$ is bounded on $[0, T], \lambda_{\max }$ is also bounded on $[0, T]$ and we denote its upper bound by $\lambda_{\text {max }}^{*}$. Then one concludes that $(8.3)$ is satisfied with $C^{0}=\lambda_{\text {max }}^{*}$, which implies that $\left(Z_{t}\right)$ is a martingale.

Proposition 8.2. Let us denote

$$
Z_{t}:=\exp \left(\int_{t}^{T} A_{s}^{T} \Sigma_{s}^{1 / 2} d W_{s}^{S}-\frac{1}{2} \int_{t}^{T}\left\|A_{s}^{T} \Sigma_{s}^{1 / 2}\right\|^{2} d s\right), \quad t \in[0, T]
$$

where $\left(A_{t}\right)_{t \in[0, T]}$ is a deterministic process with values in $\mathbb{R}^{d}$ which is bounded by $A^{*} \in \mathbb{R}^{d}$. Then $\left(Z_{t}\right)_{t \in[0, T]}$ is a martingale.

Proof. First note that it is sufficient to show that $\mathbb{E} Z_{t}=1$ for $t \in[0, T]$. Since $\left\langle W_{t, k}^{S}, W_{t, k j}^{\sigma}\right\rangle=$ $\rho_{j}$ we obtain $\left(W_{t, k}^{S}\right) \stackrel{d}{=}\left(\rho_{1} W_{t, k 1}^{\sigma}+\sqrt{1-\rho_{1}^{2}} \hat{W}_{t, k 1}\right)$ where $\left(\hat{W}_{t}\right)$ is a $d \times d$ Brownian motion matrix, independent of $\left(W_{t}^{\sigma}\right)$. Thus, when we denote $v_{\rho}:=\left(\rho_{1}, 0, \ldots, 0\right)^{T} \in \mathbb{R}^{d}$ and $\bar{v}_{\rho}:=$ $\left(\sqrt{1-\rho_{1}^{2}}, 0, \ldots, 0\right)^{T} \in \mathbb{R}^{d}$ we obtain

$$
A_{t}^{T} \Sigma_{t}^{1 / 2} d W_{t}^{S} \stackrel{d}{=} \operatorname{Tr}\left(v_{\rho} A_{t}^{T} \Sigma_{t}^{1 / 2} d W_{t}^{\sigma}\right)+\operatorname{Tr}\left(\bar{v}_{\rho} A_{t}^{T} \Sigma_{t}^{1 / 2} d \hat{W}_{t}\right)
$$

and

$$
\left\|A_{t}^{T} \Sigma_{t}^{1 / 2}\right\|^{2}=\left(\rho_{1}^{2}+\left(1-\rho_{1}^{2}\right)\right)\left\|A_{t}^{T} \Sigma_{t}^{1 / 2}\right\|^{2}=\left\|v_{\rho} A_{t}^{T} \Sigma_{t}^{1 / 2}\right\|^{2}+\left\|\bar{v}_{\rho} A_{t}^{T} \Sigma_{t}^{1 / 2}\right\|^{2} .
$$


Hence we can write

$$
\begin{aligned}
Z_{t} \stackrel{d}{=} & \exp \left(\int_{t}^{T} \operatorname{Tr}\left(v_{\rho} A_{s}^{T} \Sigma_{s}^{1 / 2} d W_{s}^{\sigma}\right)-\frac{1}{2} \int_{t}^{T}\left\|v_{\rho} A_{s}^{T} \Sigma_{s}^{1 / 2}\right\|^{2} d s\right) \\
& \cdot \exp \left(\int_{t}^{T} \operatorname{Tr}\left(\bar{v}_{\rho} A_{s}^{T} \Sigma_{s}^{1 / 2} d \hat{W}_{s}\right)-\frac{1}{2} \int_{t}^{T}\left\|\bar{v}_{\rho} A_{s}^{T} \Sigma_{s}^{1 / 2}\right\|^{2} d s\right) \\
= & \mathcal{E}\left(\int^{T} \operatorname{Tr}\left(v_{\rho} A_{s}^{T} \Sigma_{s}^{1 / 2} d W_{s}^{\sigma}\right)\right)_{t} \mathcal{E}\left(\int^{T} \operatorname{Tr}\left(\bar{v}_{\rho} A_{s}^{T} \Sigma_{s}^{1 / 2} d \hat{W}_{s}\right)\right)_{t}
\end{aligned}
$$

where $\mathcal{E}$ denotes the stochastic exponential. Now we obtain

$$
\begin{aligned}
\mathbb{E} Z_{t} & =\mathbb{E}\left[\mathbb{E}\left[Z_{t} \mid \mathcal{F}_{T}^{W^{\sigma}}\right]\right] \\
& =\mathbb{E}\left[\mathcal{E}\left(\int^{T} \operatorname{Tr}\left(v_{\rho} A_{s}^{T} \Sigma_{s}^{1 / 2} d W_{s}^{\sigma}\right)\right)_{t} \mathbb{E}\left[\mathcal{E}\left(\int^{T} \operatorname{Tr}\left(\bar{v}_{\rho} A_{s}^{T} \Sigma_{s}^{1 / 2} d \hat{W}_{s}\right)\right)_{t} \mid \mathcal{F}_{T}^{W^{\sigma}}\right]\right] .
\end{aligned}
$$

Since $\left(\hat{W}_{t}\right)$ and $\left(W_{t}^{\sigma}\right)$ are independent, the inner conditional expectation is equal to 1 due to Example 4 in Liptser \& Shiryaev (2001). From Proposition 8.1 we conclude that the remaining expression is also 1.

\section{REFERENCES}

Bru, M.-F. (1991). Wishart processes. J. Theoret. Probab. 4, 725-751.

Buraschi, A., Porchia, P. \& Trojani, F. (2010). Correlation risk and optimal portfolio choice. J. Financ. 65, 393-420.

Cont, R. \& da Fonseca, J. (2002). Dynamics of implied volatility surfaces. Quant. Finance 2, $45-60$.

Da Fonseca, J., Grasselli, M. \& Tebaldi, C. (2007). Option pricing when correlations are stochastic: An analytical framework. Rev. Deriv. Res. 10, 151-180.

Da Fonseca, J., Grasselli, M. \& Tebaldi, C. (2008). A multifactor volatility Heston model. Quant. Finance 8, 591-604.

Gnoatto, A. \& Grasselli, M. (2012). The explicit laplace transform for the Wishart process. arXiv:1107.2748 1-25.

Gourieroux, C. \& Sufana, R. (2003). Wishart quadratic term structure models. SSRN E-library $1-35$.

Gourieroux, C. \& Sufana, R. (2004). Derivative pricing with Wishart multivariate stochastic volatility: Application to credit risk. SSRN E-library 1-44.

Hata, H. \& Sekine, J. (2011). Risk-sensitive asset management with Wishart-autoregessive-type factor model. Preprint 1-30.

Heston, S. (1993). A closed-form solution for options with stochastic volatility with applications to bond and currency options. Rev. Financ. Stud. 6, 327-343.

Kallsen, J. \& Muhle-Karbe, J. (2010). Utility maximization in affine stochastic volatility models. Int. J. Theor. Appl. Finance 13, 459-477.

Korn, R. \& Kraft, H. (2004). On the stability of continuous-time portfolio problems with stochastic opportunity set. Math. Finance 14, 403-414.

Kraft, H. (2005). Optimal portfolios and Heston's stochastic volatility model: an explicit solution for power utility. Quant. Finance 5, 303-313.

Liptser, R. S. \& Shiryaev, A. N. (2001). Statistics of random processes. I, vol. 5 of Applications of Mathematics (New York). Springer-Verlag, Berlin.

Liu, J. (2007). Portfolio selection in stochastic environments. Rev. Financ. Stud. 20, 1-39.

Matsumoto, S. (2012). General moments of the inverse real Wishart distribution and orthogonal Weingarten functions. J. Theoret. Probab. 25, 798-822.

Mayerhofer, E., Pfaffel, O. \& Stelzer, R. (2011). On strong solutions for positive definite jump diffusions. Stochastic Process. Appl. 121, 2072-2086. 
Muhle-Karbe, J., Pfaffel, O. \& Stelzer, R. (2012). Option pricing in multivariate stochastic volatility models of OU type. SIAM J. Financial Math. 3, 66-94.

Rellich, F. (1969). Perturbation theory of eigenvalue problems. Gordon and Breach Science Publishers, New York.

Richter, A. (2012). Explicit solutions to quadratic BSDEs and applications to utility maximization in multivariate affine stochastic volatility models. arXiv1201.2877 1-36.

Rieder, U. \& Bäuerle, N. (2005). Portfolio optimization with unobservable Markov-modulated drift process. J. Appl. Probab. 42, 362-378.

Sultan, S. A. \& Tracy, D. S. (1996). Moments of Wishart distribution. Stochastic Anal. Appl. 14, 237-243.

Zariphopoulou, T. (2001). A solution approach to valuation with unhedgeable risks. Finance Stoch. 5, 61-82.

(N. Bäuerle) Institute for Stochastics, Karlsruhe Institute of Technology, D-76128 Karlsruhe, GERMANY

E-mail address: nicole.baeuerle@kit.edu

(Z. Li) Institute for Stochastics, Karlsruhe Institute of Technology, D-76128 Karlsruhe, GerMANY

E-mail address: zejingli@stoch.uni-karlsruhe.de 